\title{
Benign Prostatic Hyperplasia - An economic assessment of fixed combination therapy based on a literature review
}

\author{
Roberto Messina ${ }^{1}$, Vincenzo Mirone ${ }^{2}$ \\ ${ }^{1}$ National Chairman, FederAnziani Senior Italia, Italy; \\ ${ }^{2}$ General secretary of SIU - Italian Urology Society.
}

\begin{abstract}
Summary FederAnziani Senior Italia and SIU Italian Society of Urology - have decided to work together to draft a document focussing on Benign Prostatic Hyperplasia (BPH), and to stress the importance of adherence with pharmacological treatment in this setting, from both a scientific and a patient standpoint.

Starting from a literature search, the two associations analysed to what extent an increase in treatment adherence amongst these patients influences hospital savings and to what extent therapy persistence levels are affected by monotherapy rather than free drug combinations.

These estimates were performed only on patients taking medicinal products belonging to the $5 \alpha$-reductase inhibitors (5ARI) class that, although not indispensable, are the compounds that bring the greatest benefits, especially in the elderly and for which we know that every additional 30 days of therapy reduced the likelihood of acute urinary retention (AUR) and surgery by $14 \%$ and $11 \%$ respectively ".

The results show that the use of fixed combination therapy would involve an increase in persistence due to the lower rate of patients abandoning treatment over time. Each 30 day-increment of 5ARI therapy, i.e. for an expenditure of 10.6 million euros extra per year for 5ARI medication, savings of approximately 24.3 million euros in hospital costs could be achieved.
\end{abstract}

KEY WORDS: Benign prostatic hyperplasia; Alpha blockers; 5ARI drugs; Fixed combination drugs; Therapy adherencel persistence; Acute urinary retention (AUR); Lower urinary tract symptoms (LUTS).

Submitted 14 July 2015; Accepted 31 July 2015

\section{INTRODUCTION}

Treatment non-adherence can have a strong impact on health costs and on the success of the pharmacologic treatment (1). Whereas in Italy and the rest of Europe there are currently no studies on what could be the value in terms of savings obtained by better treatment adherence, in the United States it has been estimated that it may be as much as 100 billion dollars (2). The aim of this paper is to start to overcome this lack of information by proposing, in the urological setting, an economic evaluation of treatment adherence, focussing on benign prostatic hyperplasia (BPH) and highlighting the positive impact that fixed combination therapy may have on a patient's therapy persistence. The elderly consume more healthcare services than younger people and consequently require special attention in that they have special needs. These needs are quite simply the result of various factors that can include the physiological changes that take place during life, the possibility of developing several diseases, the fact that the over- 65 age class is often neglected in the development of new types of medicinal product and, last but not least, the complicated management, in everyday life, of the use of medicinal products. This latter factor can be caused by polydrug use, which, unless it is appropriately managed is likely to become a potential risk factor for poor adherence with the treatments prescribed.

As a solution to the polypharmacy issue, the World Health Organisation suggests the development and implementation of fixed dose combinations (FDC), which would help reduce the number of pills taken by the individual patients and consequently improve treatment adherence (3).

A literature search performed on a sample of 75 further literature reviews revealed how treatment adherence is the most recurrent condition for a better use of medicinal products. The other measures taken to improve adherence also include simplifying the ways in which medicinal products are taken, intended as combining the various active ingredients in the same drug (FDC - Fixed Dose Combination) $(4,5)$.

Amongst the other cases of non-adherence, the SABA Strategic Advisory Board on Adherence, like the WHO and AIFA (Italian Medicines Agency), identified the complexity deriving from polytherapy/appropriate therapy as an area of intervention for the simplification of treatments and therapeutic continuity (6).

It would therefore appear to be a paradigm recognised by the entire scientific community that a greater number of medicinal products taken is related to poorer treatment adherence (7), especially in elderly patients with cognitive impairment. Another study shows that patients taking one tablet a day have an optimum adherence of $80 \%$, a percentage that drops rapidly if subjects need to take more than one drug or need to take the same drug several times a day; if we consider that this value drops to $50 \%$ for medicinal products taken 4 times a day, it is easy to postulate that $75 \%$ of all patients and $50 \%$ of chronic patients are 
unable to follow the treatment regimen prescribed $(2,8)$. FederAnziani Senior Italia involved Agenzia Italiana del Farmaco (Italian Medicines Agency) (AIFA), Federazione Italiana dei Medici di Medicina Generale (Italian Federation of General Practitioners) and Federfarma in drawing up a joint document on the importance of treatment adherence in today's society: "Aderenza alla terapia: leva per la salute e la sostenibilità nel futuro" (Treatment adherence: a lever for health and sustainability in the future) (9).

This document identifies treatment adherence, especially when appropriate, as the first step towards a global management of patients by the main players in the healthcare supply chain: doctors, pharmacists, patients and institutions.

"FederAnziani is firmly convinced that we need to use all possible strategies to solve the problem of poor treatment adherence" (R. Messina, chairman of FederAnziani Senior Italia). It is not merely patient associations such as FederAnziani Senior Italia that are sensitive to these issues, other associations, such as some pharmacists' associations, also believe that "patient management is a priority objective of pharmacy: accompanying patients throughout their treatment programme improves treatment adherence by chronic patients. Greater adherence with therapy means more health for citizens and a better use of the public and private resources available, thereby avoiding relapses and restricting the number of re-admissions" (Annarosa Racca, chair of Federfarma) or that "for some time now, treatment adherence has been the main front in improving the population's health conditions and the sustainability of health services. Our profession has taken this pathway by implementing field trials studying how pharmacists can assist patients and general practitioners and promoting training on all levels" (Andrea Mandelli, chairman of FOFI - Federation of Italian Pharmacists Associations).

For general practitioners "generating significant growth in treatment adherence" means "increasing the efficacy of community treatment for citizens' health" [Giacomo Milillo, national secretary of the FIMMG - Federazione Italiana dei Medici di Medicina Generale (Italian General Practitioners Federation)].

As regards treatment adherence, the Ministry of Health states that the elderly are "scared they will forget to take their medicines" (Marcella Marletta, director general of the Ministry of Health's medical devices and pharmaceutical service).

Table 1.

BPH - Epidemiological situation - Italy 2014.

\section{Materials AND Methods}

This paper evaluates to what extent a one-month increase in persistence in BPH can weigh in economic terms. The analysis is conducted on $5 \alpha$-reductase inhibitors (5ARIs) alone as they are the drivers of poor adherence and for the reduction in hospitalisation, in this area of urology.

To do so, the authors first attempted to perform a literature review, where it would appear that most articles agreed in highlighting the importance of fixed combinations for adherence to treatment.

Secondly, an economic evaluation of the cost of BPH patients in Italy was obtained by multiplying the condition's prevalence rate for the various age ranges by the absolute number of male inhabitants of Italy in 2014 (most recent ISTAT data available).

The total number of patients was then multiplied by those seeking treatment (55\%) (10) and, of these, those who are effectively treated ( $78 \%$ of those seeking treatment) (11). The number of males not on treatment was estimated by subtraction. Table 1 summarises the results of this first investigation.

Compared to the total number of people being treated, we know from the pharmaceutical market (13) that the drug prescription percentages are $65 \%$ for alpha blockers (ABs), $20.11 \%$ for 5ARIs and $14.9 \%$ for free combinations; therefore, by dividing the absolute number of patients treated per age range with these percentages, we obtain the exact number of subjects taking a given type of medicinal product. In order to understand the economic value related to hospital admissions for $\mathrm{BPH}$, we calculated the hospitalisation rate without surgery of those taking 5ARI as both monotherapy (23.6 every 1,000 patients) and combination therapy (17.5 every 1,000 patients), and the hospitalisation rate with surgery (23.4 every 1,000 patients for 5ARI monotherapy and 17.3 every 1,000 patients for free combinations (14). By estimating an average cost for both surgical DRGs of $€ 3$, 126 and non-surgical diagnosis-related groups (DRG) of $€ 1,788$ (15), we estimated the hospital cost for patients treated with 5ARI as both mono- and combination therapy (CT), as being equal to 200.9 million euros (Table 2). By weighing the economic value of one-month's consumption of 5ARI in Italy, by multiplying this value by the total number of those taking 5ARIs in Italy, it was possible to estimate the total cost of one month therapy with this type of products.

The main results of this analysis are provided below.

\begin{tabular}{|c|c|c|c|c|c|c|}
\hline Age range & Prevalence $\%$ & Males in Italy & Prevalence of BPH & Patients seeking treatment & Patients treated & Not treated \\
\hline $35-44$ & $0.6 \%$ & $4,534,581$ & 27,207 & 14,964 & 11,672 & 3,292 \\
\hline $45-54$ & $4.5 \%$ & $4,742,341$ & 213,405 & 117,373 & 91,551 & 25,822 \\
\hline $55-64$ & $20.0 \%$ & $3,699,097$ & 739,819 & 406,901 & 317,383 & 89,518 \\
\hline $65-74$ & $42.0 \%$ & $3,067,166$ & $1,288,210$ & 708,515 & 552,642 & 155,873 \\
\hline $75-84$ & $51.6 \%$ & $2,008,867$ & $1,036,575$ & 570,116 & 444,691 & 125,426 \\
\hline$>=85$ & $50.3 \%$ & 601,001 & 302,304 & 166,267 & 129,688 & 36,579 \\
\hline
\end{tabular}


Table 2.

Cost of admission per type of treatment subdivided by age range. Values expressed in euros.

\begin{tabular}{|c|c|c|c|c|c|c|c|}
\hline \multirow[t]{2}{*}{ Age range } & \multicolumn{3}{|c|}{ Cost of non-surgical hospitalisation $\times 1,000$ (AUR*) } & \multicolumn{3}{|c|}{ Cost of surgical hospitalisation x $1,000 * *$} & \multirow[t]{2}{*}{ ТОТ } \\
\hline & 5ARI & CT & TOT & 5ARI & CT & TOT & \\
\hline $35-44$ & $€ 319$ & $€ 236$ & $€ 555$ & $€ 551$ & $€ 409$ & $€ 960$ & $€ 1,514,889$ \\
\hline $45-54$ & $€ 2,500$ & $€ 1,852$ & $€ 4,353$ & $€ 4,325$ & $€ 3,205$ & $€ 7,530$ & $€ 11,882,225$ \\
\hline $55-64$ & $€ 8,667$ & $€ 6,422$ & $€ 15,089$ & $€ 14,994$ & $€ 11,109$ & $€ 26,103$ & $€ 41,192,505$ \\
\hline $65-74$ & $€ 15,092$ & $€ 11,182$ & $€ 26,274$ & $€ 26,108$ & $€ 19,344$ & $€ 45,452$ & $€ 71,726,405$ \\
\hline $75-84$ & $€ 12,144$ & $€ 8,998$ & $€ 21,142$ & $€ 21,008$ & $€ 15,565$ & $€ 36,574$ & $€ 57,715,622$ \\
\hline 85 and over & $€ 3,542$ & $€ 2,624$ & $€ 6,166$ & $€ 6,127$ & $€ 4,539$ & $€ 10,666$ & $€ 16,831,998$ \\
\hline Tot & $€ 42,264$ & $€ 31,315$ & $€ 73,579$ & $€ 73,113$ & $€ 54,171$ & $€ 127,285$ & $€ 200,863,644$ \\
\hline \multicolumn{8}{|c|}{$\begin{array}{l}* \text { AUR } \rightarrow \text { ICD9CM } 788.20 \text { acute urinary retention; ICD9CM } 599.6 \text { urinary obstruction. } \\
* * \text { BPH-related surgery } \rightarrow \text { ICD9CM } 57.0 \text { bladder stone or blood clot drainage; ICD9CM } 57.91 \text { endoscopic incision of the neck of the bladder; ICD9CM } 57.94 \text { bladder catheterisation; ICD9CM } \\
60.21 \text { transurethral prostate ablation procedure; ICD9CM } 60.29 \text { transurethral prostate resection procedure; ICD9CM } 60.3 \text { adenomectomy: ICD9CM } 60.4 \text { adenomectomy } \\
\text { Source: Author's processing of data from Spatafora (10), Thales (11), Geodemo (12), IMS (13), ARNO } 2013 \text { (15), Nonis M. et al. (16]). }\end{array}$} \\
\hline
\end{tabular}

\section{Results}

Literature agrees in stating that the prevalence for $\mathrm{BPH}$ increases with age, and consequently the number of subjects affected is on the increase due to the ageing of the population $(15,17,18)$.

However, the number of subjects in whom BPH becomes symptomatic, i.e. it actually causes discomfort, is approximately half. Environmental components have been excluded from the condition's aetiology, and it is thought that a role may be played by hereditary factors, given the increased risk amongst the family members of subjects affected by the condition (19).

As is the case for all chronic illnesses, BPH treatment warrants the best results when taken constantly. The patient does not usually perceive the gradual improvements made possible by therapy as, from a subjective point of view, the disease is only considered resolved once urinary symptoms have improved. This is one of the causes, possibly the most important, of the poor persistence of treatment in BPH therapy $(19,20)$.

$66 \%$ of patients with BPH in Italy present comorbidities and consequently are treated with a number of various drugs (21).

Lower urinary tract symptoms (LUTS) determine a gradual impairment in quality of life (social relationships, leisure activities and relations with partners); however, less than $50 \%$ of men with LUTS visit their doctor, in general the first consultation of men with their doctor occurs about two years before the manifestations of symptoms, the main reasons for this being: lack of knowledge about prevention (22), embarrassment when talking about symptoms, the idea that these symptoms are "normal" and the fear of surgery $(10,19)$. This patient reluctance calls for a pro-active approach by the doctor in identifying patients with LUTS secondary to $\mathrm{BPH}$, although doctors often tend to consider BPH more a "syndrome" than a chronic invalidating condition (23). The European D-Impact study, which also involved Italy, showed that approximately 3 out of 4 patients with lower urinary tract symptoms (LUTS) who go to their general practitioners practice have an enlarged prostate. Men presenting with a greatly enlarged prostate are more likely to develop acute urinary retention (AUR) and undergo sur- gery than subjects with a smaller prostate (23). BPH also has a very high social and health-related impact: $\mathrm{BPH}$ is responsible for over 68 thousand hospital admissions per year; 35,000 of which undergo full surgery, whereas more than 32 thousand are merely admitted (24). In Italy, BPH accounts for over 320 million euros spent for pharmacological treatment and 74,834 days off work (19).

The most recent OsMed report (for 2014) indicates that the majority of patients (approximately 70\%) are treated with alpha blockers that have a prevalently symptomatic action and do not affect the natural history of the underlying condition. Approximately one third of patients is treated with 5ARIs, which by reducing the volume of the prostate, can affect the mechanisms underlying BPH and reduce disease progression and related complications. The greater prescription of alpha blockers is presumably due to the fact that the patient hopes to resolve the symptoms as quickly as possible (25).

According to current treatment options, approximately one fifth of symptomatic patients who go to their doctor will experience a gradual progression of symptoms and related discomfort with a consequent increased need for admission for surgery or Emergency Department/day hospital appointments for the introduction of a catheter to resolve situations of AUR (19).

In order to obtain a maximum benefit from pharmacological therapy, all the most important guidelines recommend, particularly in patients with a greater risk of disease progression, the use of a combination of an alpha blocker and a 5ARI $(18,26,27)$.

To provide further support to the above, we can quote the study analysing the prescription of drugs for $\mathrm{BPH}$ and the corresponding hospitalisation rates for LUTS. The results showed that over the years in which the study was conducted (2004-2008) AB and 5ARI prescriptions dropped, whereas prescriptions for CT increased by $5.4 \%$. Over the same period, both medical and surgical hospitalisation rates, per 1000 patients, related to BPH dropped, showing a greater reduction for medical DRGs than surgical ones from $4.9 \%$ in 2004 to $3.6 \%$ in 2008 and $5.5 \%$ in 2004 to $5.1 \%$ in 2008 , respectively. This shows that the use of fixed combinations would appear to be more efficient in 
the treatment of BPH-related complications (28). In general, BPH treatment persistence is approximately 30\% per year; this is related, on one hand, as mentioned previously, to patients' tendency to discontinue treatment and, on the other, to doctors' general trend of not recommending continuous chronic therapy and not monitoring $\mathrm{BPH}$ appropriately over time (29)

In one recent study on patients with newly diagnosed $\mathrm{BPH}$, it was observed that most patients suspended their BPH therapy early: about one third of patients discontinued treatment after 3 months, and $64 \%$ in one year. There are a number of reasons why patients suspend medication early: some because they felt better, some due to a lack of efficacy on symptoms, others due to the side effects (19). It goes without saying that an early discontinuation of therapy does not make it possible to obtain and maintain all the benefits for which it was prescribed.

It is interesting to note that in the $36 \%$ of patients who took medication for more than one year, the authors suggested they had a good relationship with their doctor, thanks to which patients were well informed about the risk factors of BPH progression (increased prostate volume and high PSA levels). This may have meant that patients took their condition more seriously and took the treatment for a longer period. The various treatments available for this condition achieved different adherence levels in the different patients taking them (19).

One Italian study conducted on a sample of 1.5 million male patients with $\mathrm{BPH}$ analyses the effects that monotherapy and free combination therapy (concomitant taking of two tablets) have on treatment persistence. The study states that, although the alpha blocker-5ARI combination is beneficial in the treatment of BPH in terms of both control over symptoms and disease evolution, in real life the persistence of patients treated for BPH varies according to the medicinal product taken: In particular, it was seen that patients treated with free combinations abandon therapy more frequently than patients treated with monotherapy. The same study group showed, in a previous study, that the shares of patients who continued taking treatment in the subsequent 12 months were 35\%, 18\% and 9\% respectively for alpha blockers, 5ARIs and free combination therapy. As mentioned previously, on average 29\% of patients continues therapy for at least one year. These percentages dropped in the subsequent 5 years to $15 \%, 8 \%$ and $3 \%$ respectively, i.e. $13 \%$ overall (30).

At equal conditions, there is an increase in adherence in the switch from extemporaneous combination (2 tablets) to monotherapy (1 tablet) in a potential 9\%-26\% range in the first year and 5\%-12\% in the fifth year (30).

The assumptions that are considered from here on show an improvement in both economic terms and treatment persistent terms that would be gained if fixed combination therapy were introduced instead of treatment with free combinations (3). It is plausible to suppose that the added value of fixed combination therapy is 2 -fold: the

(3) The fixed dose combination Dutasteride-Tamsulosina has already been developed and as a consequence it would help to increase adherence to therapy and provide cost savings to the National Healthcare System. However this combination is very low used in Italy due to the lack of the main condition for its use, that is reimbursement. increase in adherence obtained with the alpha blocker (35\%), which acts on symptoms; the overall efficacy of 5ARIs ( $18 \%$ adherence).

5ARIs achieve a lower hospitalisation rate but also poorer adherence; alpha blockers have a higher level of adherence because, as said previously, they are symptomatic but also have a higher hospitalisation rate; whereas CT (free combinations) show intermediate hospitalisation rates compared to those for ABs and 5ARIs, but also low adherence levels as they bring together a number of different medicinal products and therefore the patient abandons therapy more quickly (29).

In the knowledge that every 30 extra days of 5ARI treatments reduce the probability of the onset of acute urinary retention and need for surgery by $14 \%$ and $11 \%$ respectively, thereby reducing health care costs by $15 \%$ (31), by estimating the cost of one month's treatment with 5ARI in Italy (13), for both free combination therapy or monotherapy, the savings obtained by using therapy for this condition for 30 extra days were calculated.

30 extra days of treatment with 5ARIs cost the system 10.6 million euros. It is therefore possible to postulate that with a one-month increase in therapy, in one year it is possible to save approximately $€ 24.3$ million. This sum is calculated using the $14 \%$ reduction applied to the cost of non-surgical hospitalisations and the $11 \%$ reduction applied to the cost of surgical hospitalisations.

\section{Conclusions}

Literature shows that combination therapies can improve adherence levels and therefore reduce the medical costs of relapses. However, given the lack of studies on this topic, it is extremely difficult to estimate to what extent these combinations would have a positive impact on any increase in adherence. In this study, the authors merely quantified the savings obtained with 30 days of extra treatment with 5ARIS in one year, when administered both in combination and as monotherapy.

A number of critical aspects of the management of patients with BPH were also observed: these undoubtedly included patient empowerment, a correct and updated training to healthcare professionals, intented as both urologists and general practioners practitioners, that can contribute to the development of an appropriate pathway along which to guide patients.

The authors performed projections using data from countries whose healthcare systems have different characteristics, as well as different intrinsic characteristics of the populations analysed (habits, lifestyle, diet).

The estimates presented in this paper were calculated using the hospital expenditure for patients taking medication, a measurable estimate. However, it is possible to postulate that medical expenditure and the corresponding savings are greater due to the health costs incurred for patients not taking medication, but that in any case have to be hospitalised for complications related to their condition and that cannot therefore be included in the analyses. Furthermore, in order to simplify the analyses, the paper only considered the market shares of alpha blockers, 5ARIs and free combinations; all other types of drugs were intentionally excluded as the lion's share of the market is occu- 
pied by these three product types. The authors hope that the method used in this study can pave the way for the development of further studies with the aim of analysing the positive economic implications related to the correct use of medicinal products, not just in urological settings.

\section{REFERENCES}

* Oelke M, Becher K, Castro-Diaz D, et al. Appropriateness of oral drugs for long-term treatment of lower urinary tract symptoms in older persons: results of a systematic literature review and international consensus validation process (LUTS-FORTA 2014). Age Ageing. 2015 Jun 23. pii: afv077. [Epub ahead of print].

1. World Health Organization. Adherence to long-term therapies Evidence for action. 2003, pag, 3.

2. Alpert JS. The American Journal of Medicine), Compliancel Adherence to Physician-advised Diagnostic and Therapeutic Strategies. March 2014.

3. WHO - Priority for Europe and the World. A Public Health Approach to Innovation. Update on 2004 Background Paper, Written by MJC Willemen, PAF Jansen en HGM Leufkens - Background Paper 7,3 Priority Medicines for Elderly.

4. AIFA (Agenzia Italiana del Farmaco).Aderenza alle terapie e strategie per migliorare l'uso sicuro ed efficace dei farmaci. 26/08/2014.

5. Ryan R, Santesso N, Lowe D, et al. Interventions to improve safe and effective medicines use by consumers: an overview of systematic reviews. Cochrane Collaboration. The Cochrane Library 2014, Issue 4 http://onlinelibrary.wiley.com/doi/10.1002/14651858.CD007768.pub 3/abstract.

6. Corte di Giustizia Popolare per il Diritto alla Salute, Dipartimento Aderenza alle terapie, SABA - Strategic Advisory Board on Adherence, Novembre-Dicembre 2014

7. Pan F, Chernew ME, Fendrick AM. Impact of fixed-dose combination drugs on adherence to prescription medications. J Gen Intern Med. 2008; 23:611-4.

8. The European Innovation Partnership on Active and Healthy Ageing (EIP on AHA). https://webgate.ec.europa.eu/eipahal

9. Aderenza alla terapia: leva per la salute e la sostenibilità nel futuro. Documento approvato dall'Advisory Board sull'Aderenza alla Terapia (SABA - Strategic Advisory Board on Adherence) in occasione di Punto Insieme Sanità - 19 giugno 2014, In collaborazione con la Agenzia Italiana del Farmaco (AIFA) - Federazione Italiana dei Medici di Medicina Generale (FIMMG) - Federfarma.

10. Spatafora S, Conti G, Perachino M, et al. Evidence-based guidelines for the management of lower urinary tract symptoms related to uncomplicated benign prostatic hyperplasia in Italy: updated summary. Curr Med Res Opin. 2007; 23:1715-32.

11. Thales Pharmaceutical Research - CeGeDim 2011.

12. Istat - GeoDemo - Demografia in Cifre. Popolazione residente in Italia - 2015 - http://demo.istat.it/

13. IMS - Mercato Farmaceutico per IPB 2014.

14. Cindolo, et al. The effects of dutasteride and finasteride on BPHrelated hospitalization, surgery and prostate cancer diagnosis: a record-linkage analysis. Word J Urol. DOI 10,1007/s00345-0121000-4, 2012.

15. Pedrini A, Cavazzana A, Cataudella S, Rossi E. L'utilizzo dei farmaci per l'ipertrofia prostatica benigna nella pratica clinica. Osservatorio ARNO 2013. https://osservatorioarno.cineca.org/

16. Nonis M, Rosati E. Guida ai DRG ed, 2009. Manuale pratico per il corretto utilizzo della versione 24,0 del sistema CMS-DRG e della corrispondente ICD-9-CM (USA, FY 2007).
17. VIII Report Health Search - Istituto di ricerca della SIMG: Società Italiana Di Medicina Generale e delle Cure Primarie, Anno 2013/2014

18. Morlok $\mathrm{R}$, et al. Progressione clinica, ritenzione urinaria acuta, chirurgia prostatica e costi in pazienti con iperplasia prostatica benigna sottoposti a terapia di associazione precoce o ritardata con un inibitore della 5 alfa-reduttasi e un alfa-bloccante: analisi retrospettiva. Clinical Therapeutics/Volume 35, Numero 5, 2013.

19. Centro Studi SIC Sanità In Cifre - Punto Insieme Sanità - Tavolo Tecnico Urologia - 19 Giugno 2014; 25 Marzo 2015 - http://www. cortegiustiziapopolare.it/

20. Koh JS, Cho KJ, Kim HS, Kim JC. Twelve-month medication persistence in men with lower urinary tract symptoms suggestive of benign prostatic hyperplasia. J Clin Pract. 2014; 68:197-202.

21. Hutchinson A. Hutchinson A, Farmer R, et al. Characteristics of Patients Presenting with LUTS BPH in Six European Countries, European Urology 2006; 50:555-562.

22. The BPH survey: a male perspective. Market research undertaken by Kantar Healthcare, an independent market research company that specialises in medical and pharmaceutical studies; Summer 2011; 1,161 men over 50 years of age across France, Germany, Italy, Spain and the United Kingdom completed an online survey as part of the National Health and Wellness Survey. Data on file.

23. Carballido J, Fourcade R, Pagliarulo A, et al. Can benign prostatic hyperplasia be indentified in the primary care setting using only simple tests? Results of the Diagnosis IMprovement in PrimAry Care Trial, Int J Clin Pract. 2011; 65:989-96.

24. Ministero della Salute - Rapporto SDO (Schede di Dimissione Ospedaliera) 2013.

25. Osservatorio Nazionale sull'impiego dei Medicinali, L’uso dei farmaci in Italia, Rapporto Nazionale 2013, Roma: Agenzia Italiana del Farmaco, 2014.

26. SIU - Società Italiana di Urologia: Decalogo sull'utilizzo dei 5ARI nella pratica clinica: nuove evidenze e indicazioni utili, Pubblicato il 03/07/2012, http://www.siu.it/news/display/266.

27. Füllhasea C, Chappleb C, Cornuc J-N, et al. Systematic review of combination drug therapy for non-neurogenic male lower urinary tract symptoms. Eur Urol. 2013; 64:228-43.

28. Cindolo, et al. Actual medical management of lower urinary tract symptoms related to benign prostatic hyperplasia: temporal trends of prescription and hospitalization rates over 5 years in a large population of Italian men. Springer Science + Business Media Dordrecht 2013.

29. Cindolo L, Pirozzi L, Fanizza C, et al. Drug Adherence and Clinical Outcomes for Patients Under Pharmacological Therapy for Lower Urinary Tract Symptoms Related to Benign Prostatic Hyperplasia: Population-based Cohort Study. Eur Urol. 2014; 20. pii: S0302-2838(14)01180-4.

30. Cindolo, et al. Patient's adherence on pharmacological therapy for benign prostatic hyperplasia (BPH)-associated lower urinary tract symptoms (LUTS) is different: is combination therapy better than monotherapy?

31. Eaddy M1, Kruep E, Lunacsek O, Goodwin B. Establishing the clinical and economic benefits of adherence to 5-alpha reductase inhibitors in benign prostatic hyperplasia: an assessment of Medicare and Medicaid patients. Expert Opin Pharmacother. 2012; 13:2593-600.

\section{Correspondence}

Roberto Messina (Corresponding Author)

segreteria.presidenza@federanziani.it

National Chairman, FederAnziani Senior Italia, Italy

Vincenzo Mirone, MD, Professor

mirone@unina.it

General Secretary of SIU - Italian Urology Society, Italy 